\section{Acoustic radiation force impulse imaging of biopsy-proven Kikuchi disease: initial experiences for evaluating feasibility in pediatric patients}

\author{
Jeong-Min Lee', Jae-Yeon Hwang ${ }^{1,2}$, Jihyun Bae', Mae Ran Kim¹, Yong-Woo Kim', \\ Su Eun Park ${ }^{3}$ Jeong A Yeom 1 , Jieun Roh ${ }^{1}$ \\ ${ }^{1}$ Department of Radiology, Pusan National University Yangsan Hospital, Yangsan; ${ }^{2}$ Research \\ Institute for Convergence of Biomedical Science and Technology, Pusan National University \\ Yangsan Hospital, Yangsan; 'Department of Pediatrics, Pusan National University Children's \\ Hospital, Yangsan, Korea
}

Purpose: This study evaluated the feasibility of acoustic radiation force impulse (ARFI) elastography and characterized the sonographic features of lymph nodes (LNs) with Kikuchi disease in pediatric patients.

Methods: Seventy-six cervical LN biopsies were performed for the diagnosis of cervical lymphadenopathy. ARFI imaging was performed, and the characteristic ultrasound features of the biopsied LNs and the contralateral LNs were analyzed. We also reviewed clinical and conventional ultrasonographic findings.

Results: On histology, 56 patients were diagnosed with Kikuchi disease. These LNs were large and elongated, with increased perinodal echogenicity and capsular thickening. In 38 of them, ARFI elastography was performed, and the median shear wave velocity (SWV) of the biopsied LNs with Kikuchi disease $(2.19 \mathrm{~m} / \mathrm{sec}$; range, 1.45 to $4.57 \mathrm{~m} / \mathrm{sec})$ was higher than of the contralateral LNs ( $1.72 \mathrm{~m} / \mathrm{sec}$; range, 0.95 to $2.65 \mathrm{~m} / \mathrm{sec} ; \mathrm{P}<0.001)$. In patients with reactive hyperplasia, the mean SWV of the biopsied LNs $(2.00 \mathrm{~m} / \mathrm{sec}$; range, 1.49 to $2.26 \mathrm{~m} / \mathrm{sec})$ was higher than that of the contralateral LNs $(1.55 \mathrm{~m} / \mathrm{sec}$; range, 1.21 to $2.32 \mathrm{~m} / \mathrm{sec} ; \mathrm{P}=0.031)$.

Conclusion: The SWV of LNs with Kikuchi disease was significantly higher than that of the contralateral LNs. Morphologically, LNs with Kikuchi disease showed an enlarged, elongated, and oval shape, increased perinodal echogenicity, and capsular thickening. In addition to the conventional ultrasonographic findings, the application of ARFI is feasible even in pediatric patients for the evaluation of cervical lymphadenopathy.

Keywords: Elasticity imaging techniques; Histiocytic necrotizing lymphadenitis; Ultrasonography; Pediatrics

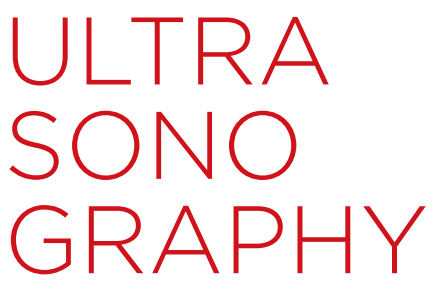

ORIGINAL ARTICLE

https://doi.org/10.14366/usg. 17067 pISSN: 2288-5919 • elSSN: 2288-5943

Ultrasonography 2019;38:58-66

Received: November 27, 2017

Revised: April 23, 2018

Accepted: April 24, 2018

Correspondence to: Jae-Yeon Hwang, MD, PhD, Department of Radiology, Pusan National University Yangsan Hospital, 20 Geumo-ro, Mulgeum-eup, Yangsan 50612, Korea

Tel. $+82-55-360-1840$

Fax. +82-55-360-1848

E-mail: jyhwang79@gmail.com

This is an Open Access article distributed under the terms of the Creative Commons Attribution NonCommercial License (http://creativecommons.org/ licenses/by-nc/3.0/) which permits unrestricted noncommercial use, distribution, and reproduction in any medium, provided the original work is properly cited.

Copyright (C) 2019 Korean Society of Ultrasound in Medicine (KSUM)

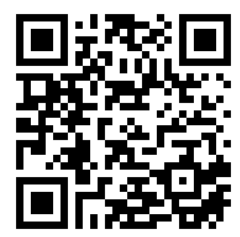

How to cite this article:

Lee JM, Hwang JY, Bae J, Kim MR, Kim YW Park SE, et al. Acoustic radiation force impulse imaging of biopsy-proven Kikuchi disease: initial experiences for evaluating feasibility in pediatric patients. Ultrasonography. 2019 Jan;38(1):58-66. 


\section{Introduction}

Cervical lymphadenopathy is a common disease in the pediatric age group. Transient reactive lymphadenopathy due to viral infection is the most common etiology. Other etiologies include systemic lupus erythematosus, Kawasaki disease, infections with the herpes simplex virus and other microorganisms, and malignancies such as nonHodgkin lymphoma, leukemia, and metastatic tumors [1-3]. In Asia, Kikuchi disease is a common cause of lymphadenopathy [4]. As recurrent episodes have been reported, it is clinically important to diagnose Kikuchi disease in order to rule out other causes of cervical lymphadenopathy $[2,3,5]$. Several reports have presented recurrence rates of $3 \%-20 \%$, although Kikuchi disease is known to be selflimiting [1-3]. Kikuchi disease can be confirmed by ultrasound (US)guided lymph node (LN) biopsy. It is characterized histopathologically by cortical and paracortical necrosis with lymphoreticular infiltrates and the absence of granulocytic infiltrates $[6,7]$.

US is a non-invasive modality of evaluating cervical lymphadenopathy. Several previous reports have described the sonographic findings of Kikuchi disease, which include elongated or oval hypoechoic LNs with increased perinodal echogenicity, an echogenic hilum, and normal hilar flow [8-11]. These features are nonspecific, and are similar to those of other common causes of lymphadenopathy, such as reactive hyperplasia, tuberculous lymphadenitis, lymphoma, and metastasis [9,12-14].

Recently, a new US-based elastography technique, acoustic radiation force impulse (ARFI) elastography, has been introduced into clinical practice. In this technique, short high-intensity acoustic pulses are emitted from the transducer and propagate shear waves in tissue. ARFI allows visual (i.e., virtual touch tissue imaging) and quantitative (i.e., virtual touch tissue quantification, which calculates the shear wave velocity) assessment of tissue stiffness. Recently, this new imaging technique has been widely applied to evaluate the cervical LNs as well as the liver, breast, thyroid, and musculoskeletal system. Several studies have reported ARFI to be effective for differentiating malignant and benign lesions in the breast and thyroid $[15,16]$.

To our best knowledge, however, no reports have described the application of ARFI in children with Kikuchi disease. The purpose of this study was to characterize the sonographic features of pediatric Kikuchi disease and to evaluate the feasibility of ARFI elastography in pediatric patients with Kikuchi disease.

\section{Materials and Methods}

\section{Study Population}

This retrospective study was approved by the institutional review board of our hospital. The requirement for informed consent from the patients was waived because this study was regarded to be of minimal risk. We reviewed the electronic medical records and histopathological reports of patients at our institution between May 2013 and April 2016. A total of 76 children $\leq 18$ years old underwent US-guided core needle biopsies for cervical lymphadenopathy. Biopsies were performed in patients with symptoms including feverassociated asymmetric lymphadenopathy with or without a rash, fatigue, or headache if those symptoms were not well controlled. Histologically, the following findings are characteristic of Kikuchi disease: a well-circumscribed paracortical focus of necrosis with a variable mixture of karyorrhectic nuclear debris, single-cell necrosis, transformed lymphocytes, and foamy macrophages [17]. Seventytwo patients were enrolled (56 with Kikuchi disease and 16 with reactive hyperplasia), excluding 4 patients who had been diagnosed with other disease (3 normal LNs, one Epstein-Barr virus infection). These patients were enrolled for an analysis of conventional US findings. For ARFI measurements, after excluding 25 patients in whom ARFI was not performed due to machine availability, a total of 47 patients (38 with Kikuchi disease and 9 with reactive hyperplasia) were enrolled for an analysis of ARFI elastography.

Age, sex, the presence and duration of fever before the US examination, and laboratory findings including leukocytosis or leukopenia, C-reactive protein levels, and the erythrocyte sedimentation rate were evaluated. Fever was defined as a single axillary temperature above $37.5^{\circ} \mathrm{C}$ or two or more measurements of a temperature above $37.2^{\circ} \mathrm{C}$. Leukocytosis was defined as an elevated white blood cell (WBC) count $\left(>10.6 \times 10^{9} / \mathrm{L}\right)$ and leukopenia was defined as a WBC count $<3.9 \times 10^{9} / \mathrm{L}$ in males and $<3.5 \times 10^{9} / \mathrm{L}$ in females. In addition, the total fever duration before and after the diagnosis, as well as the hospitalization period and whether the patient was readmitted within 1 month after discharge, were recorded to evaluate the clinical course of the enrolled patients.

\section{Conventional US}

US was performed with a dedicated US system (Acuson S2000, Siemens Medical Solutions, Erlangen, Germany and IU 22, Philips, Bothell, WA, USA) using a 9-MHz linear array transducer. All studies were conducted by a single board-certified radiologist (J.Y.H., with 11 years of experience in pediatric US). US images were retrospectively reviewed by a pediatric radiologist with 7 years of US experience and 5 years of pediatric US experience (J.B.) blinded to the final diagnosis.

On the conventional US images, the location of the LNs was identified and their diameter was measured in the long and short axes. The shape of the LNs was analyzed using the ratio of the 
shortest diameter to the longest diameter as follows: round, $>0.7$; oval, 0.5-0.7; and elongated, <0.5 [8]. Capsular thickening (a hypoechoic rim surrounding the LN), any presence of increased echogenicity of the perinodal fat tissue, internal necrosis (anechoic or markedly hypoechoic areas within the LN), and vascularity on color Doppler imaging of the target LNs were recorded. We considered increased vascularity to be present when the normal hilar blood flow and the parenchymal blood flow were shown together.

\section{Application of ARFI Measurements}

After conventional US, shear wave imaging with ARFI was performed using the same 9-MHz linear array transducer. The operator selects the $L N$ and set the depth and location of a $10 \mathrm{~mm} \times 5 \mathrm{~mm}$ region of interest (ROI), within which the shear wave velocity (SWV) $(\mathrm{m} / \mathrm{sec})$ could be determined. The propagation of the acoustic pulse varies according to tissue stiffness. During ARFI measurements, patients were required to hold their breath if they were able to cooperate. An ROI was placed in the cortex of the node in question, avoiding fatty hilum and hilar vessels as much as possible. ARFI measurements were made in the biopsied LNs and contralateral LNs located at the same level. Five measurements were performed for each LN, with the median value regarded as the representative SWV (Fig. 1) $[18,19]$.

\section{Core Needle Biopsy}

Core needle biopsies were performed using an 18-gauge dual action semiautomatic core biopsy needle (Stericut, TSK Laboratory, Tochigi, Japan) under US guidance. After local anesthesia, the needle tip was inserted into the target LN using a free-hand technique. Subsequently, the cutting cannula was fired, and at least two specimens were obtained. The biopsy specimens were fixed in formalin solution. Pathologists examined all specimens. The location of cervical lymphadenopathy was categorized into seven levels according to an imaging-based nodal classification [20].

The indications for cervical LN biopsy were as follows: lymphadenopathy of unknown cause that persisted for longer than 2-4 weeks despite adequate treatment, cervical lymphadenopathy accompanied by leukopenia or atypical clinical features other than bacterial infection, and multiple levels of adenopathy or supraclavicular adenopathy reflecting a possible increased risk of malignancy.

\section{Statistical Analysis}

A descriptive statistical analysis was conducted of demographics; the size of the LNs; clinical and laboratory findings; the location and shape of the LNs; the presence of characteristic findings, including increased perinodal echogenicity, capsular thickening, vascularity; total fever duration; hospitalization period; and readmission within 1 month after discharge. Comparisons between the Kikuchi disease group and the reactive hyperplasia group were made using the Mann-Whitney $\mathrm{U}$ test and the independent t-test.

For the SWVs of the LNs, the Shapiro-Wilk test was applied to analyze whether the data showed a normal distribution. Because the SWVs in both the Kikuchi disease and reactive hyperplasia group had a normal distribution, the independent t-test was performed to compare SWVs on the biopsied side with Kikuchi disease or reactive hyperplasia and the contralateral side. To assess the reliability of the measurements, coefficients of variation were obtained and intraobserver agreement was calculated using the intraclass correlation coefficient (ICC) for the five measured values. All statistical analyses were performed using SPSS version 23.0 (IBM Corp., Armonk, NY, USA). P-values $<0.05$ were considered to indicate statistical significance.

\section{Results}

\section{Patient Demographics and Clinical Findings}

The mean age ( \pm standard deviation [SD]) of the patients with Kikuchi disease was $12.6 \pm 3.94$ years (range, 3 to 18 years). Twenty-

Table 1. Summary of the sonographic findings of Kikuchi disease

\begin{tabular}{lccc}
\hline \multicolumn{1}{c}{ Characteristic } & $\begin{array}{c}\text { Kikuchi disease } \\
(\mathbf{n}=56)\end{array}$ & $\begin{array}{c}\text { Reactive LNs } \\
(\mathbf{n}=16)\end{array}$ & P-value \\
\hline $\begin{array}{l}\text { Long diameter }(\mathrm{cm}) \\
\text { Shape }{ }^{\text {a) }}\end{array}$ & $1.83 \pm 0.70$ & $2.26 \pm 0.93$ & 0.084 \\
$\quad$ Round (>0.7) & $5(8.9)$ & 0 & 0.493 \\
Oval (0.5-0.7) & $17(30.4)$ & $7(43.7)$ & \\
Elongated (<0.5) & $34(60.7)$ & $9(56.3)$ & \\
Location (biopsy) & & & 0.668 \\
I & $2(3.6)$ & 0 & \\
॥ & $21(37.5)$ & $6(37.5)$ & \\
III & $13(23.2)$ & $3(18.7)$ & \\
IV & $5(8.9)$ & $2(12.5)$ & \\
V & $15(26.8)$ & $5(31.3)$ & \\
Increased perinodal & $56(100)$ & $16(100)$ & 0.106 \\
echogenicity & & & \\
Capsular thickening & $49(87.5)$ & $11(68.8)$ & \\
Vascularity & & & 0.775 \\
Normal & $43(76.8)$ & $12(75.0)$ & \\
Increased & $13(23.2)$ & $4(25.0)$ & \\
Sonographic necrosis & $3(5.3)$ & $1(6.2)$ & 0.550 \\
\hline
\end{tabular}

Values are presented as mean \pm standard deviation or number (\%). LN, lymph node; SD, standard deviation.

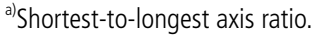




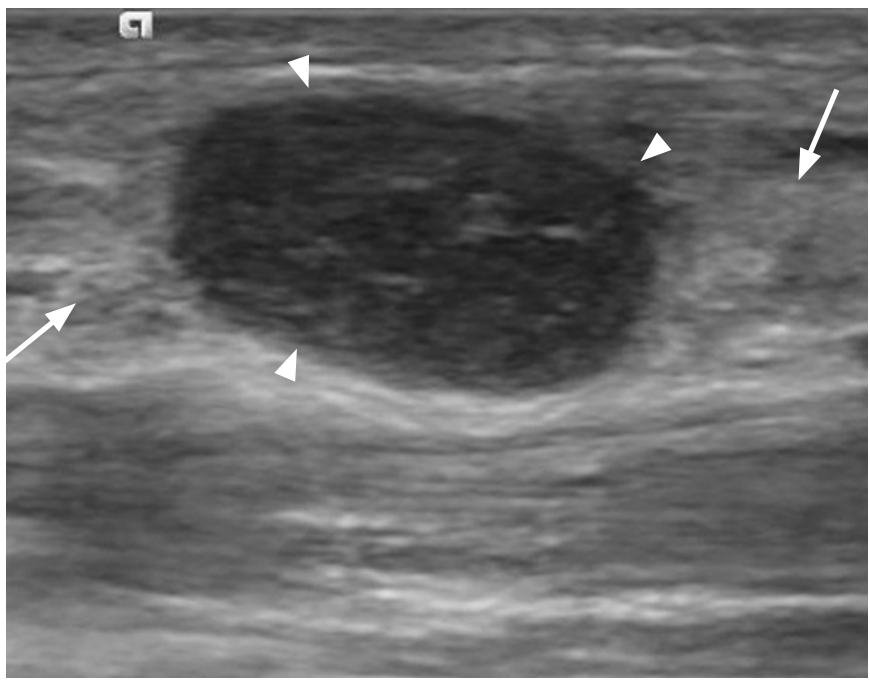

A

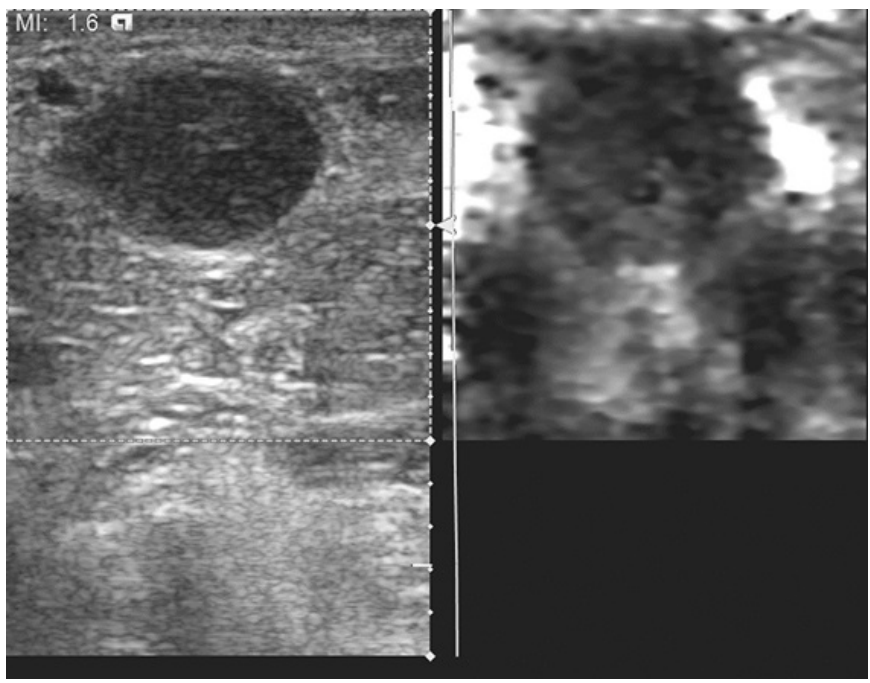

C

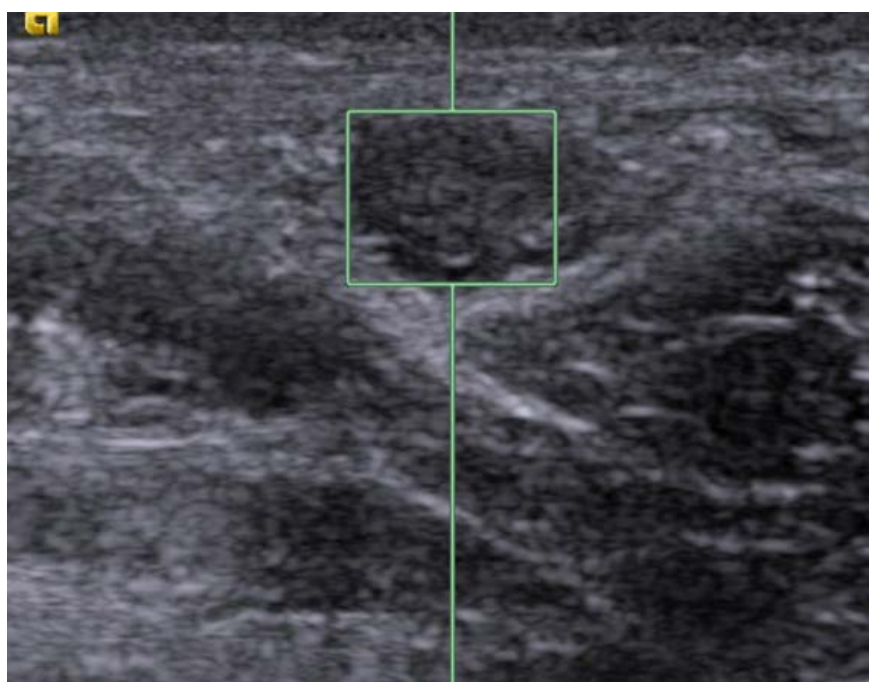

$\mathrm{E}$

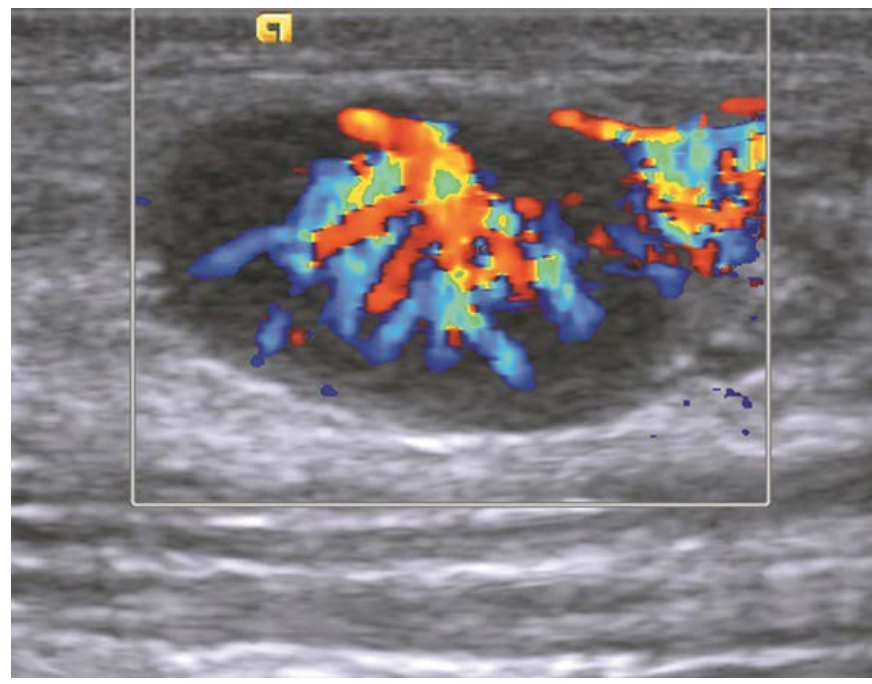

B

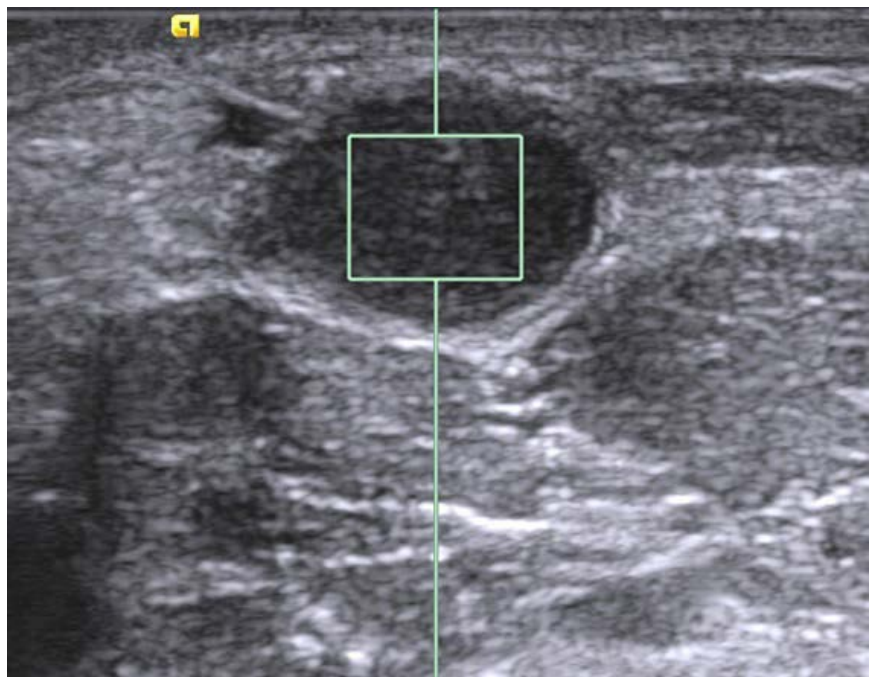

D

Fig. 1. Kikuchi disease in a 14-year-old boy.

A. On gray-scale sonography, an enlarged lymph node shows low echogenicity with increased echogenicity of the adjacent fat tissue (arrows) and capsular thickening (arrowheads) in the right neck, at level V. B. The lymph node shows increased vascularity on color Doppler imaging. C. A qualitative gray-scale map made using virtual touch tissue imaging shows hardness of the lymph node. D. In the biopsied lymph nodes, acoustic radiation force impulse (ARFI) were measured. And the median shear wave velocity (SWV) of the 5 measurements was $2.3 \mathrm{~m} / \mathrm{sec}$. E. ARFI were measured in the contralateral lymph nodes, and the median SWV of the 5 measurements was $1.3 \mathrm{~m} / \mathrm{sec}$. 
three patients were males and 33 were females. Fever was present in 44 of the 56 patients $(78.6 \%)$. The mean duration $( \pm S D)$ of fever was $13.8 \pm 6.7$ days (range, 1 to 36 days). Leukopenia was observed in 27 of 56 patients $(48.2 \%)$ and the WBC count was within the normal range in the other 29 patients (51.8\%). Leukocytosis was not observed in the patients with Kikuchi disease. The level of C-reactive protein was $1.61 \pm 1.71 \mathrm{mg} / \mathrm{dL}$ (range, 0.02 to $6.78 \mathrm{mg} / \mathrm{dL}$ ) and the erythrocyte sedimentation rate was $31.3 \pm 21.9 \mathrm{~mm} / \mathrm{hr}$ (range, 2.0 to $103.0 \mathrm{~mm} / \mathrm{hr}$ ). In contrast, of the 16 patients with reactive hyperplasia, five had leukocytosis and the other 11 had a WBC within normal range. The level of C-reactive protein was $1.28 \pm 1.65 \mathrm{mg} / \mathrm{dL}$ (range, 0.04 to $6.09 \mathrm{mg} / \mathrm{dL}$ ) and the erythrocyte sedimentation rate was $22.6 \pm 14.3 \mathrm{~mm} / \mathrm{hr}$ (range, 2.0 to 58.0 $\mathrm{mm} / \mathrm{hr}$ ). The patient demographics of the Kikuchi disease and reactive hyperplasia groups were not significantly different. The factors that showed a statistically significant difference between the Kikuchi disease and reactive hyperplasia groups were fever duration (13.8 days vs. 6.7 days, $P=0.006$ ), and the WBC count also showed a significant difference $(P<0.001)$. The other clinical findings did not show significant between-group differences.

\section{Findings of Conventional US}

The US findings of Kikuchi disease are summarized in Table 1. The mean long-axis diameter $( \pm S D)$ of the $L N s$ with Kikuchi disease was $1.83 \pm 0.70 \mathrm{~cm}$ (range, 0.73 to $3.40 \mathrm{~cm}$ ). Regarding the shape of the LNs, 34 of the 56 LNs (60.7\%) were elongated, 17 (30.4\%) were oval, and only five (8.9\%) were round. Capsular thickening was observed in 49 of the 56 patients (87.5\%) and increased perinodal echogenicity was seen in all patients. There was no internal necrosis in any patients. On color Doppler imaging, 13 of the 56 patients (23.2\%) showed increased vascularity, whereas $43(76.8 \%)$ showed normal vascularity. Core needle biopsy was most frequently performed in LNs at level II (21 of 56 patients, $37.5 \%$ ) and level V (15 of 56 patients, 26.8\%), followed by level III (13 of 56 patients, $23.2 \%$ ). No conventional US findings showed a statistically significant difference in comparison to the LNs with reactive hyperplasia $(P>0.05)$.

\section{Results of ARFI Measurements}

The results of ARFI measurements are summarized in Table 2. In the

Table 2. Shear wave velocity of LNs with Kikuchi disease and reactive hyperplasia obtained using the ARFI technique

\begin{tabular}{lcc}
\hline & Median (range, m/sec) & P-value \\
\hline Kikuchi disease $(\mathrm{n}=38)$ & $2.19(1.45-4.57)$ & $<0.001$ \\
$\quad$ Biopsy & $1.72(0.95-2.65)$ & \\
$\quad$ Contralateral & & \\
Reactive hyperplasia $(\mathrm{n}=9)$ & $2.00(1.49-2.26)$ & \\
$\quad$ Biopsy & $1.55(1.21-2.32)$ & \\
$\quad$ Contralateral &
\end{tabular}

$\mathrm{LN}$, lymph node; ARFI, acoustic radiation force impulse.

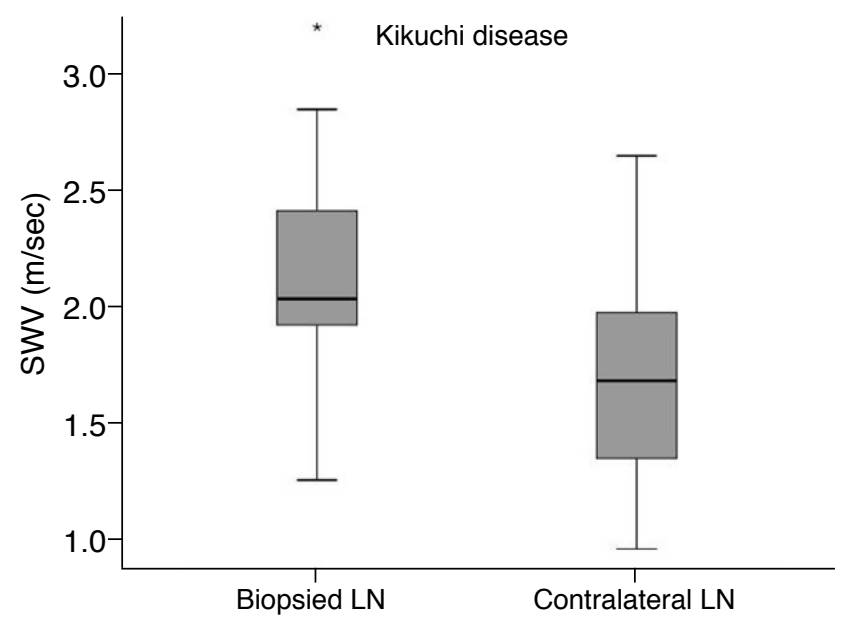

A

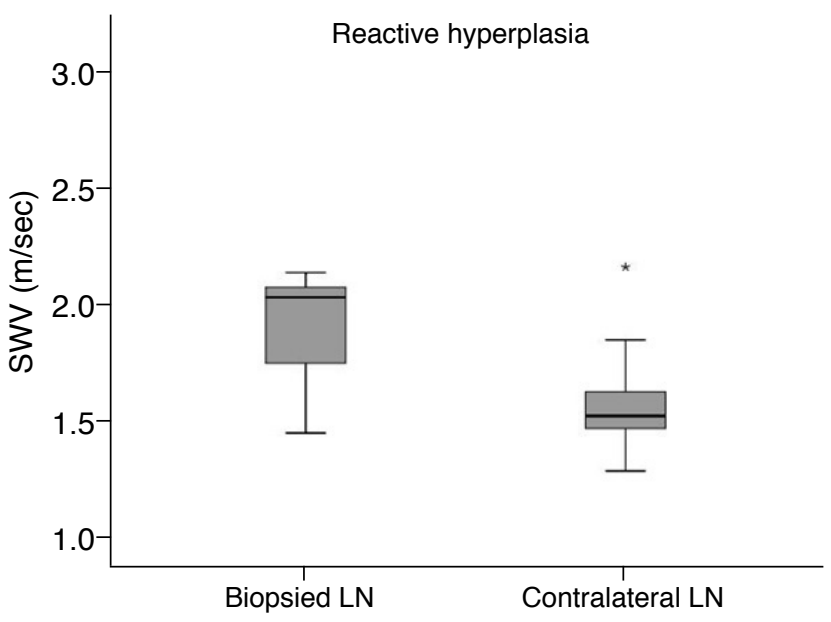

B

Fig. 2. Comparison of the shear wave velocity (SWV) in the Kikuchi disease and reactive hyperplasia.

A. In the Kikuchi disease, the median SWV of the biopsied lymph nodes (LNs) was $2.19 \mathrm{~m} / \mathrm{sec}$ and the median SWV of the contralateral LNs was $1.72 \mathrm{~m} / \mathrm{sec}$. *The outlier SWV of the biopsied LNs in the Kikuchi disease was $3.18 \mathrm{~m} / \mathrm{sec}$. B. In the reactive hyperplasia, the median SWV of the biopsied LN was $2.00 \mathrm{~m} / \mathrm{sec}$ and the median SWV of the contralateral LNs was $1.55 \mathrm{~m} / \mathrm{sec}$. *The outlier SWV of the contralateral LNs in the reactive hyperplasia was $2.24 \mathrm{~m} / \mathrm{sec}$. 


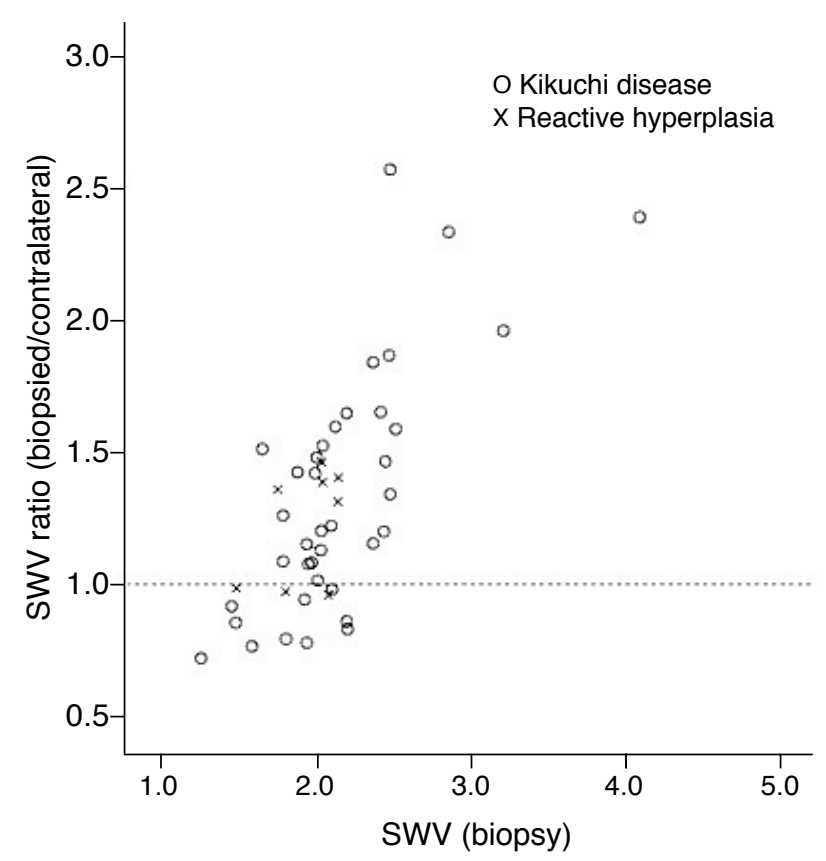

Fig. 3. Scatterplot of the ratio of the shear wave velocity (SWV) of the biopsied lymph nodes (LNs) to that of the contralateral LNs with Kikuchi disease and reactive hyperplasia. In most samples, the SWV of the biopsied LNs was higher than that of the contralateral LNs.

group with Kikuchi disease, the median SWV of the biopsied LNs was $2.19 \mathrm{~m} / \mathrm{sec}$ (range, 1.45 to $4.57 \mathrm{~m} / \mathrm{sec}$ ) and the median SWV of the contralateral LNs was $1.72 \mathrm{~m} / \mathrm{sec}$ (range, 0.95 to $2.65 \mathrm{~m} / \mathrm{sec}$ ). The SWVs of the biopsied LNs were significantly higher than those of the contralateral LNs $(P<0.001)$ (Fig. 2A). The ratio of the SWVs of the biopsied LNs to that of the contralateral LNs was 1.23 (range, 0.75 to 2.67) (Fig. 3).

In patients with reactive hyperplasia, the mean SWV of the biopsied LNs was $2.00 \mathrm{~m} / \mathrm{sec}$ (range, 1.49 to $2.26 \mathrm{~m} / \mathrm{sec}$ ) and the mean SWV of the contralateral LNs was $1.55 \mathrm{~m} / \mathrm{sec}$ (range, 1.21 to $2.32 \mathrm{~m} / \mathrm{sec}$ ) (Fig. 2B). The ratio of the SWVs of the biopsied LNs to that of the contralateral LNs was 1.29 (range, 0.85 to 1.78 ) (Fig. 3). The SWVs of the biopsied LNs were significantly higher than those of the contralateral LNs $(P=0.031)$. When we compared the SWVs of the biopsied LNs between those with Kikuchi disease and those with reactive hyperplasia, no intergroup difference was found ( $P=0.232$ ).

On average, the SWV of the biopsied LNs was higher than that of the contralateral LNs, whereas the SWV of the biopsied LNs was lower in some cases. Twelve cases showed lower SWVs in the biopsied LNs than in the contralateral LNs, of which nine were cases of Kikuchi disease and three were cases of reactive hyperplasia. The ratio of the SWVs of the biopsied LNs to those of the contralateral
LNs was 0.83 (range, 0.75 to 0.97 ) in the LNs with Kikuchi disease. In the LNs with reactive hyperplasia, the ratio of the SWVs of the biopsied LNs to that of the contralateral LNs was $0.85,0.94$, and 0.98 in each of the three cases.

The median measured depth of the biopsied LNs was $1.20 \mathrm{~cm}$ (range, 0.40 to $1.90 \mathrm{~cm}$ ) and the median measured depth of the contralateral LNs was $1.20 \mathrm{~cm}$ (range, 0.60 to $2.10 \mathrm{~cm}$ ) in the LNs with Kikuchi disease. In the reactive hyperplasia group, the median measured depth of the biopsied LNs was $0.90 \mathrm{~cm}$ (range, 0.50 to $1.70 \mathrm{~cm}$ ) and the median measured depth of the contralateral LNs was $1.25 \mathrm{~cm}$ (range, 0.40 to $1.70 \mathrm{~cm}$ ). There was no statistically significant difference between the biopsied and contralateral groups.

The intra-operator coefficients of variation for the SWVs of the biopsied and contralateral LNs were around $14 \%$ and $11 \%$, respectively. The ICC was 0.85 (95\% confidence interval [CI], 0.77 to 0.91$)$ in the biopsied LNs and $0.92(95 \% \mathrm{Cl}, 0.88$ to 0.95$)$ in the contralateral LNs.

\section{Clinical Outcomes}

The mean total fever duration $( \pm S D)$ in the patients with Kikuchi disease was $15.6 \pm 6.6$ days (range, 2.0 to 37.0 days), and the mean total fever duration $( \pm S D)$ in the patients with reactive hyperplasia was $8.8 \pm 5.9$ days (range, 2.0 to 19.0 days). The mean total fever duration was significantly longer in the Kikuchi disease group than in the reactive hyperplasia group $(P=0.004)$. The mean hospitalization periods in the Kikuchi disease and reactive hyperplasia groups were 5.1 days and 5.9 days, respectively. There was no significant difference in the hospitalization period between the two groups $(P=0.731)$. However, six of the 56 patients with Kikuchi disease were re-admitted to the hospital within 1 month. In the hyperplasia group, two of the 16 patients were re-admitted to the hospital.

\section{Discussion}

ARFI elastography is a promising noninvasive tool for the assessment of benign diseases, including Kikuchi disease, reactive hyperplasia, and tuberculosis lymphadenitis, and for distinguishing them from malignancies [14,21-23]. A study of patients aged 1192 years (mean age, 55 years) assessing 89 biopsied cervical LNs (37 benign: reactive, Kikuchi disease, tuberculosis; 52 malignant: various primary sites) with strain imaging showed a high sensitivity for differentiating benign from malignant LNs, with a sensitivity of $98.1 \%$ for the strain ratio and $88.4 \%$ for the elasticity score [14].

Our study showed that the SWV of the biopsied LNs with Kikuchi disease was significantly higher than that of the contralateral LNs by roughly 1.35 times (mean), even though Kikuchi disease is benign. 
Morphologically, the LNs involved in Kikuchi disease demonstrate architecture effaced by paracortical expansion composed of circumscribed foci of apoptotic necrosis with abundant karyorrhectic debris and numerous histiocytes of different types at the edge of the necrotic foci [24]. Depending on the time course, karyorrhexis or a mononuclear reaction with variable cellular composition may predominate [25]. Therefore, we hypothesize that the increased SWV of Kikuchi disease may be caused by an increase in the cellularity of the LNs combined with periadenitis and associated soft tissue swelling.

However, there was no intergroup difference between the LNs with Kikuchi disease and those with reactive hyperplasia. For similar reasons to those relevant for Kikuchi disease, reactive LNs become enlarged due to hyperplasia of the cellular components, which are mixed small and large lymphocytes and numerous tangible body macrophages with no cytologic atypia in cases of reactive hyperplasia [26]. Therefore, the SWV of reactive LNs reflects a higher density than that of normal LNs. Moreover, in a recent study, Kikuchi disease was found to demonstrate a soft appearance on strain elastography, suggestive of a benign lesion, and there were no statistically significant differences between Kikuchi disease and other benign diseases $[27,28]$. This finding suggests that it may be difficult to differentiate Kikuchi disease from reactive hyperplasia with only shear wave elastography.

Several studies have reported unilateral neck involvement to be predominant in Kikuchi disease, occurring in up to $80 \%$ of cases $[6,11]$. Based on previous research, we assumed that patients had unilateral disease. However, in a few cases, the biopsied LNs unexpectedly showed lower SWV values than the contralateral LNs. About 24\% (9 of 38) patients with Kikuchi disease showed a lower SWV in the biopsied LNs than in the contralateral LNs. In seven of these nine cases, the contralateral LNs were enlarged and showed perinodal infiltration and capsular thickening, similarly to the biopsied LNs. In addition, the median SWV ratio of the biopsied LNs to the contralateral LNs was 0.86 (range, 0.75 to 0.97 ), indicating that the SWVs of the bilateral LNs were not significantly different. In these cases, it is considered that Kikuchi disease probably affected the bilateral cervical LNs. In addition, the contact and pressure applied on the neck of the patient via the operator's hand could alter the measurements. In the shear wave elastography technique, the elasticity of the structures is influenced by the external pressure applied, and the stiffness rises with increasing pressure due to the nonlinear elastic effect artifact [29]. We hypothesize that this discordant result may have been due to technical errors while obtaining SWV values.

Generally, the major concern with US examinations is variability due to high operator dependency, especially for quantitative elastography measurements. Although US was performed by a single radiologist in our study, the ICC values of the SWVs were excellent in both the biopsied LNs and the contralateral LNs. Compared with several other methods, ARFI imaging showed higher contrast transfer efficacy, resulting in greater reproducibility and less interoperator variability [30]. The reproducibility of the ARFI technique has been found to be fair to excellent for evaluating cervical LNs, ranging from 0.64 to 0.96 for the intra-observer ICC and from 0.72 to 0.77 for the inter-observer ICC $[30,31]$. Kikuchi disease has various clinical features, ranging from an asymptomatic presentation to severe systemic symptoms such as prolonged fever and arthralgia. No diagnostic clinical findings are available to confirm the diagnosis of Kikuchi disease, but leukopenia is a consistent laboratory finding $[32,33]$. In this study, leukopenia was present in about $50 \%$ of patients with Kikuchi disease, whereas leukopenia was not present in the reactive hyperplasia group. All patients with reactive hyperplasia showed leukocytosis or a normal WBC count.

The sonographic findings of LNs with Kikuchi disease are variable, as they can show either benign or malignant features, including reactive hyperplasia, tuberculous lymphadenitis, lymphoma, and metastatic LNs $[8,34]$. The usual sonographic findings of LNs with Kikuchi disease are unilateral enlargement of multiple LNs and increased perinodal echogenicity $[1,34]$. LNs with Kikuchi disease show an elongated or oval shape, hypoechogenicity of the nodal cortex with an echogenic hilum, and normal hilar flow [8]. Another study of the sonographic features of Kikuchi disease found that conglomerated unilateral cervical lymphadenopathy with perinodal fat swelling and an even size distribution were common findings. In that study, the mean size of the LNs was $1.6 \mathrm{~cm}$ and the LNs were mainly located at levels II and V $[9,11]$.

Our study showed US findings similar to those of previous studies in terms of size, shape, location of LNs, and vascularity on color Doppler imaging $[8,10]$. In our study, the LNs with Kikuchi disease were enlarged, with a mean diameter of $1.83 \mathrm{~cm}$. The LNs were mostly elongated, and an oval shape was found in $91.1 \%$ (51 of 56). Only five LNs were round. In a comparative study of benign and malignant LNs, malignant LNs tended to be round (shortest-tolongest axis ratio $>0.5$ ), while benign LNs tended to be oval $[35,36]$. In the present study, 49 biopsies were performed of LNs located at levels II, III, and V. Increased vascularity was observed in 13 LNs with Kikuchi disease $(23.2 \%)$, which is a higher prevalence than has been observed in previous studies $(4.4 \%-7.4 \%)[8,10]$.

We also found increased perinodal echogenicity and capsular thickening in both the Kikuchi disease and reactive hyperplasia groups. Benign LNs in Kikuchi disease and reactive hyperplasia may have increased perinodal echogenicity and diffuse capsular thickening $[37,38]$. Increased perinodal echogenicity, also known 
as periadenitis, takes place when structures surrounding the LNs are infiltrated by perivascular and interstitial inflammatory cells, including a mixture of lymphoid cells and histiocytes and the characteristic karyorrhectic debris [39]. Although the sonographic findings revealed that LNs affected by Kikuchi disease tended to be probably benign, some LNs with Kikuchi disease can show findings suspicious for malignancy, such as a round shape, loss of the central fatty hilum, and eccentric capsular thickening [8]. Therefore, there are limitations in the application of gray-scale sonography alone to differentiate Kikuchi disease.

The clinical course of Kikuchi disease showed a longer duration of fever than that of reactive hyperplasia. Six of the 56 patients were re-admitted to the hospital within 1 month. The main symptoms leading to re-admission were fever, leukopenia, or headache. Of particular note, in three cases, a poor prognosis with meningoencephalitis accompanied by complications of Kikuchi disease was seen. In the hyperplasia group, two of the 16 patients were re-admitted to the hospital for tonsillectomy with treatment of tonsillitis. As above, the prognosis was worse in patients with Kikuchi disease than in those with reactive hyperplasia.

There were several limitations in this study. First, this was a retrospective study, and thus the evaluation of the gray-scale images and color Doppler images was limited. In addition, for this reason, the ability to calculate failure rates in SWV measurements was limited. Second, the SWV measurements were made by a single radiologist, so we could not assess inter-observer reproducibility. Third, we assumed that patients had unilateral disease. Previous studies have reported unilateral neck involvement to be predominant in Kikuchi disease, occurring in up to $80 \%$ of cases $[6,11]$. In the remaining $20 \%$, LN biopsy was performed at the site of any symptoms or abnormalities on both sides. Therefore, the other side is not always normal, and it is possible that the bilateral LNs were affected by the disease. Finally, we did not analyze the differences between the Kikuchi disease group or the reactive hyperplasia group and a group with malignant disease. Since malignant cervical lymphadenopathy with lymphadenopathy, the causes of which include Hodgkin lymphoma, leukemia, and metastasis, is rarely seen in children (1.15\%) [40], it was not easy to enroll malignant lesions in our study.

In conclusion, the SWV of LNs in Kikuchi disease was significantly higher than that of the contralateral LNs. Although no significant difference was found between Kikuchi disease and reactive hyperplasia, which are benign diseases, the application of ARFI elastography was confirmed to be feasible, even in pediatric patients, for evaluating cervical lymphadenopathy. Morphologically, LNs with Kikuchi disease showed enlargement, an elongated and oval shape, increased perinodal echogenicity, and capsular thickening, similarly to LNs affected by other benign diseases. Further studies with more robust data and a wider range of diseases will demonstrate the promise of US elastography for application in pediatric patients.

ORCID: Jeong-Min Lee: https://orcid.org/0000-0002-7675-6045; Jae-Yeon Hwang: https://orcid.org/0000-0003-2777-3444; Jihyun Bae: https://orcid.org/0000-00032143-6308; Mae Ran Kim: https://orcid.org/0000-0001-8648-5763; Yong-Woo Kim https://orcid.org/0000-0002-1703-7753; Su Eun Park: https://orcid.org/0000-00015860-821X; Jeong A Yeom: https://orcid.org/0000-0002-0328-7989; Jieun Roh: https://orcid.org/0000-0002-6876-3990

\section{Conflict of Interest}

No potential conflict of interest relevant to this article was reported.

\section{References}

1. Han HJ, Lim GY, Yeo DM, Chung NG. Kikuchi's disease in children: clinical manifestations and imaging features. J Korean Med Sci 2009;24:1105-1109.

2. Song JY, Lee J, Park DW, Sohn JW, Suh SI, Kim IS, et al. Clinical outcome and predictive factors of recurrence among patients with Kikuchi's disease. Int J Infect Dis 2009;13:322-326.

3. Tsang WY, Chan JK, Ng CS. Kikuchi's lymphadenitis: a morphologic analysis of 75 cases with special reference to unusual features. Am J Surg Pathol 1994;18:219-231.

4. Lin HC, Su CY, Huang SC. Kikuchi's disease in Asian children. Pediatrics 2005;115:e92-e96.

5. Blewitt RW, Kumar SN, Abraham JS. Recurrence of Kikuchi's lymphadenitis after 12 years. J Clin Pathol 2000;53:157-158.

6. Kwon SY, Kim TK, Kim YS, Lee KY, Lee NJ, Seol HY. CT findings in Kikuchi disease: analysis of 96 cases. AJNR Am J Neuroradiol 2004;25:1099-1102.

7. Onciu M, Medeiros LJ. Kikuchi-Fujimoto lymphadenitis. Adv Anat Pathol 2003;10:204-211.

8. Yoo JL, Suh SI, Lee YH, Seo HS, Kim KM, Shin BK, et al. Gray scale and power Doppler study of biopsy-proven Kikuchi disease. J Ultrasound Med 2011:30:957-963.

9. Ying M, Ahuja AT, Yuen HY. Grey-scale and power Doppler sonography of unusual cervical lymphadenopathy. Ultrasound Med Biol 2004;30:449-454.

10. Ryoo I, Suh S, Lee YH, Seo HS, Seol HY. Comparison of ultrasonographic findings of biopsy-proven tuberculous lymphadenitis and Kikuchi disease. Korean J Radiol 2015;16:767775.

11. Kim JY, Lee H, Yun B. Ultrasonographic findings of Kikuchi cervical lymphadenopathy in children. Ultrasonography 2017;36:66-70.

12. Bosch $X$, Guilabert A, Miquel R, Campo E. Enigmatic KikuchiFujimoto disease: a comprehensive review. Am J Clin Pathol 2004;122:141-152.

13. Koybasi S, Saydam L, Gungen Y. Histiocytic necrotizing 
lymphadenitis of the neck. Am J Otolaryngol 2003;24:344-347.

14. Alam F, Naito K, Horiguchi J, Fukuda H, Tachikake T, Ito K. Accuracy of sonographic elastography in the differential diagnosis of enlarged cervical lymph nodes: comparison with conventional B-mode sonography. AJR Am J Roentgenol 2008;191:604-610.

15. Xu JM, Xu XH, Xu HX, Zhang YF, Zhang J, Guo LH, et al. Conventional US, US elasticity imaging, and acoustic radiation force impulse imaging for prediction of malignancy in thyroid nodules. Radiology 2014;272:577-586.

16. Magalhaes M, Belo-Oliveira P, Casalta-Lopes J, Costa Y, Goncalo M, Gomes P, et al. Diagnostic value of ARFI (Acoustic Radiation Force Impulse) in differentiating benign from malignant breast lesions. Acad Radiol 2017;24:45-52.

17. O'Malley DP, George TI, Orazi A, Abbondanzoz SL. Atlas of nontumor pathology: benign and reactive conditions of lymph node and spleen. Washington, DC: Amer Registry of Pathology, 2009.

18. Bhatia KS, Cho CC, Tong CS, Yuen EH, Ahuja AT. Shear wave elasticity imaging of cervical lymph nodes. Ultrasound Med Biol 2012;38:195-201.

19. Mjelle AB, Mulabecirovic A, Hausken T, Havre RF, Gilja OH, Vesterhus $M$. Ultrasound and point shear wave elastography in livers of patients with primary sclerosing cholangitis. Ultrasound Med Biol 2016;42:2146-2155.

20. Som PM, Curtin HD, Mancuso AA. Imaging-based nodal classification for evaluation of neck metastatic adenopathy. AJR Am J Roentgenol 2000;174:837-844.

21. Meng W, Xing $P$, Chen Q, Wu C. Initial experience of acoustic radiation force impulse ultrasound imaging of cervical lymph nodes. Eur J Radiol 2013;82:1788-1792.

22. Zhai L, Palmeri ML, Bouchard RR, Nightingale RW, Nightingale KR. An integrated indenter-ARFI imaging system for tissue stiffness quantification. Ultrason Imaging 2008;30:95-111.

23. Teng DK, Wang $H$, Lin $Y Q$, Sui GQ, Guo F, Sun LN. Value of ultrasound elastography in assessment of enlarged cervical lymph nodes. Asian Pac J Cancer Prev 2012;13:2081-2085.

24. Hutchinson CB, Wang E. Kikuchi-Fujimoto disease. Arch Pathol Lab Med 2010;134:289-293.

25. Pilichowska ME, Pinkus JL, Pinkus GS. Histiocytic necrotizing lymphadenitis (Kikuchi-Fujimoto disease): lesional cells exhibit an immature dendritic cell phenotype. Am J Clin Pathol 2009;131:174182.

26. Slack GW. The Pathology of reactive lymphadenopathies: a discussion of common reactive patterns and their malignant mimics. Arch Pathol Lab Med 2016;140:881-892.
27. Lee KH, Ryu J. Real-time elastography of cervical lymph nodes in Kikuchi disease. J Ultrasound Med 2014;33:2201-2205.

28. Choi YJ, Lee JH, Baek JH. Ultrasound elastography for evaluation of cervical lymph nodes. Ultrasonography 2015;34:157-164.

29. Sigrist RMS, Liau J, Kaffas AE, Chammas MC, Willmann JK. Ultrasound elastography: review of techniques and clinical applications. Theranostics 2017;7:1303-1329.

30. Bhatia K, Tong CS, Cho CC, Yuen EH, Lee J, Ahuja AT. Reliability of shear wave ultrasound elastography for neck lesions identified in routine clinical practice. Ultraschall Med 2012;33:463-468.

31. Cheng KL, Choi YJ, Shim WH, Lee JH, Baek JH. Virtual touch tissue imaging quantification shear wave elastography: prospective assessment of cervical lymph nodes. Ultrasound Med Biol 2016;42:378-386.

32. Chuang $\mathrm{CH}$, Yan DC, Chiu CH, Huang YC, Lin PY, Chen CJ, et al. Clinical and laboratory manifestations of Kikuchi's disease in children and differences between patients with and without prolonged fever. Pediatr Infect Dis J 2005;24:551-554.

33. Kuo TT. Kikuchi's disease (histiocytic necrotizing lymphadenitis): a clinicopathologic study of 79 cases with an analysis of histologic subtypes, immunohistology, and DNA ploidy. Am J Surg Pathol 1995; 19:798-809.

34. Fulcher AS. Cervical lymphadenopathy due to Kikuchi disease: US and CT appearance. J Comput Assist Tomogr 1993;17:131-133.

35. Ahuja AT, Ying $M$, Ho SY, Antonio $G$, Lee $Y P$, King AD, et al. Ultrasound of malignant cervical lymph nodes. Cancer Imaging 2008:8:48-56.

36. Ying $M$, Bhatia KS, Lee YP, Yuen HY, Ahuja AT. Review of ultrasonography of malignant neck nodes: greyscale, Doppler, contrast enhancement and elastography. Cancer Imaging 2014;13:658-669.

37. Asano $S$, Akaike $Y$, Muramatsu T, Wakasa $H$, Yoshida $H$, Kondou $R$, et al. Necrotizing lymphadenitis: a clinicopathological and immunohistochemical study of four familial cases and five recurrent cases. Virchows Arch A Pathol Anat Histopathol 1991;418:215-223.

38. Restrepo R, Oneto J, Lopez K, Kukreja K. Head and neck lymph nodes in children: the spectrum from normal to abnormal. Pediatr Radiol 2009;39:836-846.

39. Li YY, Chang WC, Hsu YP, Liao LJ. Ultrasound features of pediatric Kikuchi-Fujimoto disease: report of two cases. J Med Ultrasound 2011;19:87-90.

40. Niedzielska G, Kotowski M, Niedzielski A, Dybiec E, Wieczorek P. Cervical lymphadenopathy in children: incidence and diagnostic management. Int J Pediatr Otorhinolaryngol 2007;71:51-56. 